\title{
Necessary and sufficient conditions for functions involving the psi function to be completely monotonic
}

\author{
Zhen-Hang Yang, Yu-Ming Chu* and Xiao-Hui Zhang
}

"Correspondence:

chuyuming2005@126.com School of Mathematics and

Computation Science, Hunan City University, Yiyang, 413000, China

\begin{abstract}
We present the necessary and sufficient conditions such that the functions involving $R(x)=\psi(x+1 / 2)-\ln x$ with a parameter are completely monotonic on $(0, \infty)$, find three new sequences which are fast convergence toward the Euler-Mascheroni constant, and give a positive answer to the conjecture proposed by Chen (J. Math. Inequal. 3(1):79-91, 2009), where $\psi$ is the digamma function.
\end{abstract}

MSC: 33B15;26D15

Keywords: psi function; completely monotone function; Euler-Mascheroni constant

\section{Introduction}

A real-valued function $f$ is said to be completely monotonic on the interval $I$ if $f$ has derivatives of all orders on $I$ and satisfies

$$
(-1)^{n} f^{(n)}(x) \geq 0
$$

for all $x \in I$ and $n=0,1,2, \ldots f$ is said to be strictly completely monotonic on $I$ if inequality (1.1) is strict.

It is well known that $f$ is completely monotonic on $(0, \infty)$ if and only if

$$
f(x)=\int_{0}^{\infty} e^{-x t} d \mu(t)
$$

where $\mu$ is a nonnegative measure on $[0, \infty)$ such that the integral is convergent for all $x>0$ (see [1], p.161).

Let $x>0$, then the classical Euler gamma function $\Gamma$ and psi (digamma) function $\psi$ are, respectively, defined by

$$
\Gamma(x)=\int_{0}^{\infty} t^{x-1} e^{-t} d t, \quad \psi(x)=\frac{\Gamma^{\prime}(x)}{\Gamma(x)}
$$

The derivatives $\psi^{\prime}, \psi^{\prime \prime}, \psi^{\prime \prime \prime}, \ldots$ are known as polygamma functions. Recently, the gamma and polygamma functions have attracted the attention of many researchers since they play

(c) 2015 Yang et al.; licensee Springer. This article is distributed under the terms of the Creative Commons Attribution 4.0 International License (http://creativecommons.org/licenses/by/4.0/), which permits unrestricted use, distribution, and reproduction in any medium, provided you give appropriate credit to the original author(s) and the source, provide a link to the Creative Commons license, and indicate if changes were made. 
important roles in many branches, such as mathematical physics, probability, statistics, and engineering.

Let $H_{n}=\sum_{k=1}^{n} \frac{1}{k}$ be the harmonic number and $D_{n}=H_{n}-\ln n$. Then the well-known Euler-Mascheroni constant $\gamma=0.577215664 \ldots$ can be expressed as $\gamma=H_{n}-\psi(n+1)$ or $\gamma=\lim _{n \rightarrow \infty} D_{n}$, and the double inequality

$$
\frac{1}{2(n+1)}<D_{n}-\gamma<\frac{1}{2 n}
$$

holds for all $n \in \mathbb{N}$ (see [2,3]). Therefore, the convergence rate of $D_{n}$ is very slowly. Recently, many results involving the quicker convergence toward the Euler-Mascheroni constant can be found in the literature [4-27].

In 1993, DeTemple [7] introduced the DeTemple sequence

$$
R_{n}=\sum_{k=1}^{n} \frac{1}{k}-\ln \left(n+\frac{1}{2}\right)
$$

and found that it satisfies the double inequalities

$$
\frac{1}{24(n+1)^{2}}<R_{n}-\gamma<\frac{1}{24 n^{2}}
$$

and

$$
\frac{7}{960} \frac{1}{(n+1)^{4}}<R_{n}-\gamma-\frac{1}{24 n^{2}}<\frac{7}{960} \frac{1}{n^{4}}
$$

for all $n \in \mathbb{N}$.

Villarino ([14], Theorem 1.7) proved that the double inequality

$$
\frac{1}{24(n+1 / 2)^{2}+21 / 5}<R_{n}-\gamma<\frac{1}{24(n+1 / 2)^{2}+1 /(1-\ln 3+\ln 2-\gamma)-54}
$$

holds for all $n \in \mathbb{N}$ with the best possible constants $21 / 5$ and $1 /(1-\ln 3+\ln 2-\gamma)-54=$ $3.739 \ldots$.

In [18], Chen proved that the double inequality

$$
\frac{1}{24}(n+\lambda)^{-2}<R_{n}-\gamma<\frac{1}{24}\left(n+\frac{1}{2}\right)^{-2}
$$

holds for all $n \in \mathbb{N}$ with the best possible constants

$$
\lambda=\frac{1}{2 \sqrt{6(1-\gamma-\ln 3+\ln 2)}}-1=0.551 \ldots
$$

and $1 / 2$.

Mortici ([28], Theorem 2.1) presented the bounds for $R_{n}-\gamma$ as follows:

$$
\frac{1}{24}\left(n+\frac{1}{2}+\frac{7}{80 n}\right)^{-2}<R_{n}-\gamma<\frac{1}{24}\left(n+\frac{1}{2}\right)^{-2} .
$$


In [29-31], the authors established the inequality

$$
\gamma+\ln \left(n+\frac{1}{2}\right)<\sum_{k=1}^{n} \frac{1}{k} \leq \gamma+\ln \left(n+e^{1-\gamma}-1\right)
$$

which is equivalent to

$$
0<R_{n}-\gamma \leq \ln \frac{n+e^{1-\gamma}-1}{n+1 / 2} .
$$

Karatsuba [32] proved that the sequence

$$
H(n)=\left(R_{n}-\gamma\right) n^{2}=\left(\psi(n+1)-\ln \left(n+\frac{1}{2}\right)\right) n^{2}
$$

is strictly increasing with respect to all $n \in \mathbb{N}$.

In [33], the authors pointed out that $(1+1 / n)^{2} H(n)$ is a strictly decreasing and convex sequence by use of computer experiments. Chen ([15], Theorem 2) proved that both $H(n)$ and $((n+1 / 2) / n)^{2} H(n)$ are strictly increasing and concave sequences, while $((n+1) / n)^{2} H(n)$ is a strictly decreasing and convex sequence, and conjectured that:

(i) The two functions $H(x)=[\psi(x+1)-\ln (x+1 / 2)] x^{2}$ and $[(x+1 / 2) / x]^{2} H(x)$ are so-called Bernstein functions on $(0, \infty)$. That is,

$$
\begin{aligned}
& H(x)>0, \quad(-1)^{n}[H(x)]^{(n+1)}>0, \\
& ((x+1 / 2) / x)^{2} H(x)>0, \quad(-1)^{n}\left[((x+1 / 2) / x)^{2} H(x)\right]^{(n+1)}>0
\end{aligned}
$$

for $x>0$ and $n \in \mathbb{N}$.

(ii) The function $((x+1) / x)^{2} H(x)$ is strictly completely monotonic on $(0, \infty)$.

It is not difficult to verify that

$$
\begin{aligned}
& -H^{\prime \prime}\left(0^{+}\right)=2 \ln 2-2 \gamma=-0.2318 \ldots<0, \\
& -H^{\prime \prime}(1 / 2)=2 \gamma+4 \ln 2+\frac{7}{2} \sum_{n=1}^{\infty} \frac{1}{n^{3}}-\pi^{2}+\frac{7}{4}=0.01461 \ldots>0 .
\end{aligned}
$$

Therefore, the function $H(x)$ is not a Bernstein function on $(0, \infty)$.

The main purpose of this paper is to give a positive answer to the conjecture (ii) and present several necessary and sufficient conditions such that the functions involving

$$
R(x)=\psi(x+1 / 2)-\ln x
$$

with a parameter are strictly completely monotone on $(0, \infty)$.

\section{Lemmas}

In order to prove our results we need several lemmas, which we present in this section.

Lemma 1 Let $R(x)$ be defined by $(1.10)$ and $Q(t)$ be defined on $(0, \infty)$ by

$$
Q(t)=\frac{1}{t}-\frac{1}{2 \sinh \frac{t}{2}}
$$


Then the following identities are valid:

$$
\begin{aligned}
& R(x)=\int_{0}^{\infty} e^{-x t} Q(t) d t, \\
& x R(x)=\int_{0}^{\infty} e^{-x t} Q^{\prime}(t) d t, \\
& x^{2} R(x)=\frac{1}{24}+\int_{0}^{\infty} e^{-x t} Q^{\prime \prime}(t) d t, \\
& x^{3} R(x)=\frac{1}{24} x+\int_{0}^{\infty} e^{-x t} Q^{\prime \prime \prime}(t) d t, \\
& x^{4} R(x)=\frac{1}{24} x^{2}-\frac{7}{960}+\int_{0}^{\infty} e^{-x t} Q^{(4)}(t) d t .
\end{aligned}
$$

Proof Making use of the integral representations [34], p.259

$$
\psi(x)=\int_{0}^{\infty}\left(\frac{e^{-t}}{t}-\frac{e^{-x t}}{1-e^{-t}}\right) d t \text { and } \ln x=\int_{0}^{\infty} \frac{e^{-t}-e^{-x t}}{t} d t .
$$

we get

$$
\begin{aligned}
R(x) & =\psi\left(x+\frac{1}{2}\right)-\ln x=\int_{0}^{\infty}\left(\frac{e^{-x t}}{t}-\frac{e^{-(x+1 / 2) t}}{1-e^{-t}}\right) d t \\
& =\int_{0}^{\infty} e^{-x t}\left(\frac{1}{t}-\frac{1}{2 \sinh \frac{t}{2}}\right) d t=\int_{0}^{\infty} e^{-x t} Q(t) d t .
\end{aligned}
$$

Integration by parts leads to

$$
\begin{aligned}
x R(x) & =x \int_{0}^{\infty} e^{-x t} Q(t) d t=-\int_{0}^{\infty} Q(t) d e^{-x t} \\
& =-\left.e^{-x t} Q(t)\right|_{0} ^{\infty}+\int_{0}^{\infty} e^{-x t} Q^{\prime}(t) d t=\int_{0}^{\infty} e^{-x t} Q^{\prime}(t) d t,
\end{aligned}
$$

where the last equality holds due to $\lim _{t \rightarrow \infty}\left(e^{-x t} Q(t)\right)=\lim _{t \rightarrow 0}\left(e^{-x t} Q(t)\right)=0$.

Integration by parts again together with

$$
\lim _{t \rightarrow \infty}\left(e^{-x t} Q^{\prime}(t)\right)=0 \quad \text { and } \quad \lim _{t \rightarrow 0}\left(e^{-x t} Q^{\prime}(t)\right)=-\frac{1}{24}
$$

leads to

$$
\begin{aligned}
x^{2} R(x) & =x \int_{0}^{\infty} e^{-x t} Q^{\prime}(t) d t=-\left.e^{-x t} Q^{\prime}(t)\right|_{0} ^{\infty}+\int_{0}^{\infty} e^{-x t} Q^{\prime \prime}(t) d t \\
& =\frac{1}{24}+\int_{0}^{\infty} e^{-x t} Q^{\prime \prime}(t) d t .
\end{aligned}
$$

Similarly, we have

$$
\begin{aligned}
x^{3} R(x) & =\frac{1}{24} x+x \int_{0}^{\infty} e^{-x t} Q^{\prime \prime}(t) d t=\frac{1}{24} x-\left.e^{-x t} Q^{\prime \prime}(t)\right|_{0} ^{\infty}+\int_{0}^{\infty} e^{-x t} Q^{\prime \prime \prime}(t) d t \\
& =\frac{1}{24} x+\int_{0}^{\infty} e^{-x t} Q^{\prime \prime \prime}(t) d t
\end{aligned}
$$


due to $\lim _{t \rightarrow \infty}\left(e^{-x t} Q^{\prime \prime}(t)\right)=\lim _{t \rightarrow 0}\left(e^{-x t} Q^{\prime \prime}(t)\right)=0$, and

$$
\begin{aligned}
x^{4} R(x) & =\frac{1}{24} x^{2}+x \int_{0}^{\infty} e^{-x t} Q^{\prime \prime \prime}(t) d t=\frac{1}{24} x^{2}-\left.e^{-x t} Q^{\prime \prime \prime}(t)\right|_{0} ^{\infty}+\int_{0}^{\infty} e^{-x t} Q^{(4)}(t) d t \\
& =\frac{1}{24} x^{2}-\frac{7}{960}+\int_{0}^{\infty} e^{-x t} Q^{(4)}(t) d t
\end{aligned}
$$

due to

$$
\lim _{t \rightarrow \infty}\left(e^{-x t} Q^{\prime \prime \prime}(t)\right)=0 \quad \text { and } \quad \lim _{t \rightarrow 0}\left(e^{-x t} Q^{\prime \prime \prime}(t)\right)=-\frac{7}{960} .
$$

Lemma 2 ([35], Lemma 7) Let $P(t)$ be a power series which is convergent on $(0, \infty)$ defined by

$$
P(t)=\sum_{i=m+1}^{\infty} a_{i} t^{i}-\sum_{i=0}^{m} a_{i} t^{i}
$$

where $a_{i} \geq 0$ and $a_{j} \geq 0$ for $i \geq m+1$ and $0 \leq j \leq m-1, a_{m}>0$, and $\sum_{i=m+1}^{\infty} a_{i}>0$. Then there exists $t_{0} \in(0, \infty)$ such that $P\left(t_{0}\right)=0, P(t)<0$ for $t \in\left(0, t_{0}\right)$ and $P(t)>0$ for $t \in\left(t_{0}, \infty\right)$.

Lemma 3 Let $Q(t)$ be defined by (2.1). Then $Q^{\prime}(t) \geq c_{0} Q(t)$ for $t>0$, where

$$
c_{0}=\min _{t>0}\left(\frac{Q^{\prime}(t)}{Q(t)}\right)=-0.06187 \ldots
$$

Proof Simple computations lead to

$$
\begin{aligned}
& \frac{Q^{\prime}(t)}{Q(t)}=\frac{\frac{1}{4} \frac{\cosh \frac{t}{2}}{\sinh ^{2} \frac{t}{2}}-\frac{1}{t^{2}}}{\frac{1}{t}-\frac{1}{2 \sinh \frac{t}{2}}}=\frac{t^{2} \cosh \frac{t}{2}-4 \sinh ^{2} \frac{t}{2}}{4 t \sinh ^{2} \frac{t}{2}-2 t^{2} \sinh \frac{t}{2}}, \\
& \left(\frac{Q^{\prime}(t)}{Q(t)}\right)^{\prime}=\frac{1}{4 t^{2}\left(t-2 \sinh \frac{t}{2}\right)^{2} \sinh ^{2} \frac{t}{2}}\left(-16 t \sinh ^{3} \frac{t}{2}+t^{4} \cosh ^{2} \frac{t}{2}+2 t^{3} \sinh ^{3} \frac{t}{2}\right. \\
& \left.\quad-t^{4} \sinh ^{2} \frac{t}{2}+16 \sinh ^{4} \frac{t}{2}+8 t^{2} \cosh \frac{t}{2} \sinh ^{2} \frac{t}{2}-4 t^{3} \cosh ^{2} \frac{t}{2} \sinh \frac{t}{2}\right) \\
& :=\frac{p\left(\frac{t}{2}\right)}{4 t^{2}\left(t-2 \sinh \frac{t}{2}\right)^{2} \sinh ^{2} \frac{t}{2}},
\end{aligned}
$$

where

$$
\begin{aligned}
p(t)= & 16\left(t^{4} \cosh ^{2} t+t^{3} \sinh ^{3} t-t^{4} \sinh ^{2} t+\sinh ^{4} t-2 t \sinh ^{3} t\right. \\
& \left.+2 t^{2} \cosh t \sinh ^{2} t-2 t^{3} \cosh ^{2} t \sinh t\right) .
\end{aligned}
$$

Using the 'product into sum' formulas and Taylor expansion we get

$$
\begin{aligned}
\frac{1}{2} p(t)= & \cosh 4 t+4 t^{2} \cosh 3 t-2 t^{3} \sinh 3 t-4 t \sinh 3 t-4 \cosh 2 t \\
& -4 t^{2} \cosh t-10 t^{3} \sinh t+12 t \sinh t+8 t^{4}+3
\end{aligned}
$$




$$
\begin{aligned}
= & \sum_{n=0}^{\infty} \frac{4^{2 n} t^{2 n}}{(2 n) !}+4 \sum_{n=1}^{\infty} \frac{3^{2 n-2} t^{2 n}}{(2 n-2) !}-2 \sum_{n=2}^{\infty} \frac{3^{2 n-3} t^{2 n}}{(2 n-3) !}-4 \sum_{n=1}^{\infty} \frac{3^{2 n-1} t^{2 n}}{(2 n-1) !} \\
& -4 \sum_{n=0}^{\infty} \frac{2^{2 n} t^{2 n}}{(2 n) !}-4 \sum_{n=1}^{\infty} \frac{t^{2 n}}{(2 n-2) !}-10 \sum_{n=2}^{\infty} \frac{t^{2 n}}{(2 n-3) !}+12 \sum_{n=1}^{\infty} \frac{t^{2 n}}{(2 n-1) !}+8 t^{4}+3 \\
:= & \sum_{n=3}^{\infty} \frac{u_{n}}{(2 n) !} t^{2 n}
\end{aligned}
$$

where

$$
u_{n}=4^{2 n}-8 n\left(2 n^{2}-9 n+13\right) 3^{2 n-3}-2^{2 n+2}-8 n\left(10 n^{2}-13 n+1\right) .
$$

It is not difficult to verify that $u_{3}=0, u_{n}<0$ for $4 \leq n \leq 10$ and $u_{11}=1,636,643,754,240>$ 0 . Note that

$$
\begin{aligned}
u_{n+1}-16 u_{n}= & 8\left(14 n^{3}-117 n^{2}+199 n-54\right) 3^{2 n-3}+48 \times 2^{2 n} \\
& +1,200 n^{3}-1,800 n^{2}+88 n+16>0
\end{aligned}
$$

for $n \geq 11$. Therefore, $u_{n} \geq 0$ for $n \geq 11$.

From Lemma 2 we clearly see that there exists $t_{0} \in(0, \infty)$ such that $Q^{\prime}(t) / Q(t)$ is strictly decreasing on $\left(0, t_{0}\right)$ and strictly increasing on $\left(t_{0}, \infty\right)$. Therefore, Lemma 3 follows from the piecewise monotonicity of $Q^{\prime} / Q$ and the numerical computations results $t_{0}=15.4015 \ldots$ and $Q^{\prime}\left(t_{0}\right) / Q\left(t_{0}\right)=-0.06187 \ldots$

\section{Lemma 4 The inequalities}

$$
\begin{aligned}
& \frac{\sinh t}{t}>3 \frac{2 \cosh t+3}{\cosh t+14} \\
& \frac{\sinh t}{t}>15 \frac{2 \cosh ^{2} t+10 \cosh t+9}{2 \cosh ^{2} t+101 \cosh t+212} \\
& \frac{\sinh t}{t}<15 \frac{18 \cosh ^{2} t+160 \cosh t+179}{1,159 \cosh ^{2} t+4,192 \cosh t+4} \cosh t
\end{aligned}
$$

hold for $t>0$.

Proof Inequality (2.8) can be found in [36], Theorem 18.

To prove (2.9), it suffices to show that for $t>0$,

$$
p_{1}(t):=\frac{2 \cosh ^{2} t+101 \cosh t+212}{2 \cosh ^{2} t+10 \cosh t+9} \sinh t-15 t>0 .
$$

Simple computations lead to

$$
\begin{aligned}
p_{1}^{\prime}(t) & =\frac{2 \cosh ^{2} t+101 \cosh t+212}{2 \cosh ^{2} t+10 \cosh t+9} \cosh t-7 \frac{26 \cosh ^{2} t+116 \cosh t+173}{\left(2 \cosh ^{2} t+10 \cosh t+9\right)^{2}} \sinh ^{2} t-15 \\
& =\frac{4(\cosh t-1)^{5}}{\left(2 \cosh ^{2} t+10 \cosh t+9\right)^{2}}>0
\end{aligned}
$$

which implies that $p_{1}(t)>p_{1}(0)=0$. 
Similarly, inequality (2.10) is equivalent to

$$
p_{2}(t):=\frac{1,159 \cosh ^{2} t+4,192 \cosh t+4}{\left(18 \cosh ^{2} t+160 \cosh t+179\right) \cosh t} \sinh t-15 t<0 .
$$

Differentiating $p_{2}(t)$ yields

$$
\begin{aligned}
p_{2}^{\prime}(t)= & \frac{1,159 \cosh ^{2} t+4,192 \cosh t+4}{\left(18 \cosh ^{2} t+160 \cosh t+179\right) \cosh t} \cosh t \\
& +\left(\sinh ^{2} t\right) \frac{d}{d x}\left(\frac{1,159 x^{2}+4,192 x+4}{\left(18 x^{2}+160 x+179\right) x}\right)-15 \\
= & -\frac{4(1,215 x+179)(x-1)^{5}}{x^{2}\left(18 x^{2}+160 x+179\right)^{2}}<0,
\end{aligned}
$$

where $x=\cosh t>1$. Therefore, $p_{2}(t)<p_{2}(0)=0$.

Lemma 5 Let $Q(t)$ be defined by (2.1). Then the inequality

$$
q_{1}(t):=Q^{\prime \prime}(t)+\frac{7}{40} Q(t)>0
$$

holds for all $t>0$.

Proof Simple computations lead to

$$
\begin{aligned}
& Q^{\prime \prime}(t)=\frac{1}{8 \sinh \frac{t}{2}}-\frac{1}{4} \frac{\cosh ^{2} \frac{t}{2}}{\sinh ^{3} \frac{t}{2}}+\frac{2}{t^{3}} \\
& q_{1}(t)=-\frac{1}{80} \frac{20 t^{3} \cosh ^{2} \frac{t}{2}-14 t^{2} \sinh ^{3} \frac{t}{2}-3 t^{3} \sinh ^{2} \frac{t}{2}-160 \sinh ^{3} \frac{t}{2}}{t^{3} \sinh ^{3} \frac{t}{2}} .
\end{aligned}
$$

Making use of inequality (2.8) we get

$$
\begin{aligned}
\left(80 \sinh ^{3} t\right) q_{1}(2 t)= & 20\left(\frac{\sinh t}{t}\right)^{3}+7\left(\sinh ^{2} t\right) \frac{\sinh t}{t} \\
& +3 \sinh ^{2} t-20 \cosh ^{2} t \\
> & 20\left(3 \frac{2 \cosh t+3}{\cosh t+14}\right)^{3}+7\left(\cosh ^{2} t-1\right)\left(3 \frac{2 \cosh t+3}{\cosh t+14}\right) \\
& +3\left(\cosh ^{2} t-1\right)-20 \cosh ^{2} t \\
= & 25\left(\cosh ^{2} t+24 \cosh t+240\right) \frac{(\cosh t-1)^{3}}{(\cosh t+14)^{3}}>0 .
\end{aligned}
$$

Lemma 6 Let $Q(t)$ be defined by (2.1). Then

$$
q_{2}(t):=Q^{(4)}(t)-\frac{31}{336} Q(t)<0
$$

for all $t>0$. 
Proof Simple computations lead to

$$
\begin{aligned}
Q^{(4)}(t)= & \frac{7}{8} \frac{\cosh ^{2} \frac{1}{2} t}{\sinh ^{3} \frac{1}{2} t}-\frac{3}{4} \frac{\cosh ^{4} \frac{1}{2} t}{\sinh ^{5} \frac{1}{2} t}-\frac{5}{32 \sinh \frac{1}{2} t}+\frac{24}{t^{5}} \\
q_{2}(t)= & -\frac{1}{336} \\
& \times \frac{252 t^{5} \cosh ^{4} \frac{t}{2}+31 t^{4} \sinh ^{5} \frac{t}{2}+37 t^{5} \sinh ^{4} \frac{t}{2}-8,064 \sinh ^{5} \frac{t}{2}-294 t^{5} \cosh ^{2} \frac{t}{2} \sinh ^{2} \frac{t}{2}}{t^{5} \sinh ^{5} \frac{t}{2}} \\
-\left(672 \sinh ^{5} t\right) q_{2}(2 t)= & -504\left(\frac{\sinh t}{t}\right)^{5}+31\left(\sinh ^{4} t\right) \frac{\sinh t}{t} \\
& +504 \cosh ^{4} t-588 \cosh ^{2} t \sinh ^{2} t+74 \sinh ^{4} t
\end{aligned}
$$

Let

$$
U(y)=-504 y^{5}+31\left(\sinh ^{4} t\right) y+504 \cosh ^{4} t-588 \cosh ^{2} t \sinh ^{2} t+74 \sinh ^{4} t .
$$

Then it suffices to prove that $U((\sinh t) / t)>0$ for $t>0$.

It follows from $U^{\prime}(y)=31 \sinh ^{4} t-2,520 y^{4}$ that $U$ is strictly increasing with respect to $y$ on $(1, \sqrt[4]{31 / 2,520} \sinh t]$ and strictly decreasing with respect to $y$ on $[\sqrt[4]{31 / 2,520} \sinh t, \infty)$. We divide the proof into two cases.

Case 1: $t \in(\sqrt[4]{2,520 / 31}, \infty)$. Then inequality (2.8) leads to

$$
1<3 \frac{2 \cosh t+3}{\cosh t+14}<\frac{\sinh t}{t}<\sqrt[4]{\frac{31}{2,520}} \sinh t
$$

that is,

$$
3 \frac{2 \cosh t+3}{\cosh t+14}, \frac{\sinh t}{t} \in(1, \sqrt[4]{31 / 2,520} \sinh t)
$$

and so

$$
\begin{aligned}
U\left(\frac{\sinh t}{t}\right)> & U\left(3 \frac{2 \cosh t+3}{\cosh t+14}\right) \\
= & \left(504 \cosh ^{4} t-588 \cosh ^{2} t \sinh ^{2} t+74 \sinh ^{4} t\right) \\
& +31\left(\sinh ^{4} t\right) \times 3 \frac{2 \cosh t+3}{\cosh t+14}-504\left(3 \frac{2 \cosh t+3}{\cosh t+14}\right)^{5} .
\end{aligned}
$$

Let $\cosh t=x$, then $\sinh ^{2} t=x^{2}-1$, and

$$
U\left(\frac{\sinh t}{t}\right)>\frac{(x-1)^{3}}{(x+14)^{5}} U_{1}(x)
$$

where

$$
\begin{aligned}
U_{1}(x)= & 176 x^{6}+10,523 x^{5}+245,869 x^{4}+2,810,864 x^{3} \\
& +12,467,224 x^{2}+12,511,688 x-20,756,344 .
\end{aligned}
$$


It is not difficult to verify that $U_{1}(x)>U_{1}(1)=7,290,000>0$, which implies that $U((\sinh t) / t)>0$ for $t \in(\sqrt[4]{2,520 / 31}, \infty)$.

Case 2: $t \in(0, \sqrt[4]{2,520 / 31}]$. Then it follows from (2.10) and the piecewise monotonicity of $U$ that

$$
\begin{aligned}
& \infty>15 \frac{18 \cosh ^{2} t+160 \cosh t+179}{1,159 \cosh ^{2} t+4,192 \cosh t+4} \cosh t>\frac{\sinh t}{t}>\sqrt[4]{\frac{31}{2,520}} \sinh t, \\
& U\left(\frac{\sinh t}{t}\right)>U\left(15 \frac{18 \cosh ^{2} t+160 \cosh t+179}{1,159 \cosh ^{2} t+4,192 \cosh t+4} \cosh t\right) .
\end{aligned}
$$

Let $\cosh t=x$, then

$$
U\left(\frac{\sinh t}{t}\right)>U\left(15 \frac{18 x^{2}+160 x+179}{1,159 x^{2}+4,192 x+4} x\right)=\frac{(x-1)^{4}}{\left(1,159 x^{2}+4,192 x+4\right)^{5}} U_{2}(x),
$$

where

$$
\begin{aligned}
U_{2}(x)= & 14,379,675,269,523,570 x^{11}+357,214,567,270,415,330 x^{10} \\
& +3,604,910,878,299,956,955 x^{9}+19,027,526,850,473,930,600 x^{8} \\
& +55,570,610,110,726,848,080 x^{7}+85,295,682,448,077,545,696 x^{6} \\
& +54,079,668,524,631,977,864 x^{5}+560,130,320,580,220,160 x^{4} \\
& +1,016,873,963,329,280 x^{3}+923,378,178,560 x^{2} \\
& +418,677,504 x+75,776>0 .
\end{aligned}
$$

Lemma 7 Let $Q(t)$ be defined by (2.1). Then

$$
q_{3}(t):=Q^{(4)}(t)+\frac{11,165}{8,284} Q^{\prime \prime}(t)+\frac{199,849}{1,391,712} Q(t)>0
$$

for all $t>0$.

Proof It follows from (2.1), (2.11), and (2.12) that

$$
\begin{aligned}
q_{3}(t)= & \frac{1}{2,783,424 t^{5} \sinh ^{5} \frac{t}{2}}\left(-2,087,568 t^{5} \cosh ^{4} \frac{t}{2}+1,497,636 t^{5} \cosh ^{2} \frac{t}{2} \sinh ^{2} \frac{t}{2}\right. \\
& -165,829 t^{5} \sinh ^{4} \frac{t}{2}+399,698 t^{4} \sinh ^{5} \frac{t}{2} \\
& \left.+7,502,880 t^{2} \sinh ^{5} \frac{t}{2}+66,802,176 \sinh ^{5} \frac{t}{2}\right), \\
\left(2,783,424 \sinh ^{5} t\right) q_{3}(2 t) & -\left(2,087,568 \cosh ^{4} t+165,829 \sinh ^{4} t-1,497,636 \cosh ^{2} t \sinh ^{2} t\right) \\
& +199,849\left(\sinh ^{4} t\right) \frac{\sinh t}{t}+937,860\left(\sinh ^{2} t\right)\left(\frac{\sinh t}{t}\right)^{3} \\
& +2,087,568\left(\frac{\sinh t}{t}\right)^{5} .
\end{aligned}
$$


Let $\cosh t=x>1$, then (2.9) leads to

$$
\begin{aligned}
\left(2,783,424 \sinh ^{5} t\right) q_{3}(2 t)> & -\left(2,087,568 x^{4}+165,829\left(x^{2}-1\right)^{2}-1,497,636 x^{2}\left(x^{2}-1\right)\right) \\
& +199,849\left(x^{2}-1\right)^{2}\left(15 \frac{2 x^{2}+10 x+9}{2 x^{2}+101 x+212}\right) \\
& +937,860\left(x^{2}-1\right)\left(15 \frac{2 x^{2}+10 x+9}{2 x^{2}+101 x+212}\right)^{3} \\
& +2,087,568\left(15 \frac{2 x^{2}+10 x+9}{2 x^{2}+101 x+212}\right)^{5} \\
= & \frac{7(x-1)^{5}}{\left(2 x^{2}+101 x+212\right)^{5}} q_{4}(x)>0,
\end{aligned}
$$

where the last inequality holds due to

$$
\begin{aligned}
q_{4}(x)= & 10,249,024 x^{9}+2,015,594,800 x^{8}+163,876,520,192 x^{7} \\
& +6,681,271,280,040 x^{6}+136,012,433,414,956 x^{5} \\
& +1,069,481,086,377,851 x^{4}+4,121,483,475,973,500 x^{3} \\
& +8,450,810,874,059,188 x^{2}+8,899,895,239,232,240 x \\
& +3,802,278,457,617,584 .
\end{aligned}
$$

\section{Main results}

Theorem 1 Let $R(x)$ be defined on $(0, \infty)$ by (1.10). Then the function

$$
h_{a}(x)=(x+a)^{2} R(x)
$$

is strictly completely monotonic on $(0, \infty)$ if $a \geq a_{0}=\sqrt{c_{0}^{2}+7 / 40}-c_{0}=0.4847 \ldots$, where $c_{0}=-0.06187 \ldots$ is given by $(2.7)$.

Proof It follows from (2.2)-(2.4) that

$$
\begin{aligned}
h_{a}(x) & =(x+a)^{2} R(x)=x^{2} R(x)+2 a x R(x)+a^{2} R(x) \\
& =\frac{1}{24}+\int_{0}^{\infty} e^{-x t} Q^{\prime \prime}(t) d t+2 a \int_{0}^{\infty} e^{-x t} Q^{\prime}(t) d t+a^{2} \int_{0}^{\infty} e^{-x t} Q(t) d t \\
& =\frac{1}{24}+\int_{0}^{\infty} e^{-x t}\left(Q^{\prime \prime}(t)+2 a Q^{\prime}(t)+a^{2} Q(t)\right) d t \\
& \triangleq \frac{1}{24}+\int_{0}^{\infty} e^{-x t} Q(t) \delta_{a}(t) d t .
\end{aligned}
$$

We clearly see that $Q(t)>0$ for $t>0$ and Lemmas 3 and 5 imply that

$$
\begin{aligned}
\delta_{a}(t) & =\frac{Q^{\prime \prime}(t)}{Q(t)}+2 a \frac{Q^{\prime}(t)}{Q(t)}+a^{2} \geq a^{2}+2 a c_{0}-\frac{7}{40} \\
& =\left(a+c_{0}+\sqrt{c_{0}^{2}+\frac{7}{40}}\right)\left(a+c_{0}-\sqrt{c_{0}^{2}+\frac{7}{40}}\right) \geq 0
\end{aligned}
$$

if $a \geq \sqrt{c_{0}^{2}+7 / 40}-c_{0}$ 
Taking $a=1 / 2$ and replacing $x$ by $(x+1 / 2)$ in Theorem 1 , we have the following.

Corollary 1 The function $((x+1) / x)^{2} H(x)$ is strictly completely monotonic on $(-1 / 2, \infty)$.

Remark 1 Corollary 1 gives a positive answer to the conjecture (ii) posed by Chen in [15].

Theorem 2 Let $R(x)$ be defined on $(0, \infty)$ by $(1.10)$. Then the function

$$
x \mapsto F_{a}(x)=24\left(x^{2}+a\right) R(x)-1
$$

is strictly completely monotonic on $(0, \infty)$ if and only if $a \geq a_{1}=7 / 40$.

Proof The necessity follows from

$$
\lim _{x \rightarrow \infty} \frac{F_{a}(x)}{x^{-2}}=\lim _{x \rightarrow \infty} \frac{24\left(x^{2}+a\right)(\psi(x+1 / 2)-\ln x)-1}{x^{-2}}=a-\frac{7}{40} \geq 0 .
$$

It follows from (2.2) and (2.4) that

$$
\begin{aligned}
F_{a}(x) & =24\left(x^{2}+a\right) R(x)-1=24 x^{2} R(x)+24 a R(x)-1 \\
& =24\left(\frac{1}{24}+\int_{0}^{\infty} e^{-x t} Q^{\prime \prime}(t) d t\right)+24 a \int_{0}^{\infty} e^{-x t} Q(t) d t-1 \\
& =24 \int_{0}^{\infty} e^{-x t}\left(Q^{\prime \prime}(t)+a Q(t)\right) d t .
\end{aligned}
$$

From Lemma 5 we clearly see that

$$
Q^{\prime \prime}(t)+a Q(t) \geq Q^{\prime \prime}(t)+\frac{7}{40} Q(t)>0
$$

for $t>0$ if $a \geq 7 / 40$.

Note that

$$
\begin{aligned}
& F_{7 / 40}\left(n+\frac{1}{2}\right)=24\left((n+1 / 2)^{2}+7 / 40\right) R_{n}-1, \\
& F_{7 / 40}(3 / 2)=\frac{286}{5}-\frac{291}{5} \ln \frac{3}{2}-\frac{291}{5} \gamma=0.00797 \ldots, \quad F_{7 / 40}(\infty)=0 .
\end{aligned}
$$

Therefore, we have the following.

Corollary 2 Let $R_{n}$ be defined by (1.3). Then the double inequality

$$
\frac{1}{24\left((n+1 / 2)^{2}+7 / 40\right)}<R_{n}-\gamma<\frac{1+\lambda_{1}}{24\left((n+1 / 2)^{2}+7 / 40\right)}
$$

holds for $n \in \mathbb{N}$ with the best possible constants $\lambda_{1}=F_{7 / 40}(3 / 2)=0.00797 \ldots$.

Theorem 3 Let $R(x)$ be defined on $(0, \infty)$ by $(1.10)$. Then the function

$$
x \mapsto f_{a}(x)=-24\left(x^{4}+a\right) R(x)+x^{2}-\frac{7}{40}
$$

is strictly completely monotonic on $(0, \infty)$ if and only if $a \leq a_{2}=-31 / 336$. 
Proof The necessity can be deduced by

$$
\lim _{x \rightarrow \infty} \frac{f_{a}(x)}{x^{-2}}=\lim _{x \rightarrow \infty} \frac{-24\left(x^{4}+a\right)(\psi(x+1 / 2)-\ln x)+x^{2}-\frac{7}{40}}{x^{-2}}=-a-\frac{31}{336} \geq 0 .
$$

It follows from (2.2), (2.4), and (2.6) that

$$
\begin{aligned}
f_{a}(x) & =-24 x^{4} R(x)-24 a R(x)+x^{2}-\frac{7}{40} \\
& =-24\left(\frac{1}{24} x^{2}-\frac{7}{960}+\int_{0}^{\infty} e^{-x t} Q^{(4)}(t) d t\right)-24 a \int_{0}^{\infty} e^{-x t} Q(t) d t+x^{2}-\frac{7}{40} \\
& =24 \int_{0}^{\infty} e^{-x t}\left(-Q^{(4)}(t)-a Q(t)\right) d t .
\end{aligned}
$$

From Lemma 6 we clearly see that

$$
-Q^{(4)}(t)-a Q(t) \geq-Q^{(4)}(t)+\frac{31}{336} Q(t)>0
$$

if $a \leq a_{2}=-31 / 336$.

Making use of the monotonicity of $f_{a_{2}}$ and the facts that

$$
f_{a_{2}}\left(\frac{3}{2}\right)=\frac{835}{7} \gamma+\frac{835}{7} \ln \frac{3}{2}-\frac{32,819}{280}=0.009063 \ldots, \quad f_{a_{2}}(\infty)=0
$$

we have the following.

Corollary 3 Let $R_{n}$ be defined by (1.3). Then the double inequality

$$
\frac{1}{24} \frac{\left(n+\frac{1}{2}\right)^{2}-\frac{7}{40}-\lambda_{2}}{\left(n+\frac{1}{2}\right)^{4}-\frac{31}{336}}<R_{n}-\gamma<\frac{1}{24} \frac{\left(n+\frac{1}{2}\right)^{2}-\frac{7}{40}}{\left(n+\frac{1}{2}\right)^{4}-\frac{31}{336}}
$$

holds for $n \in \mathbb{N}$ with the best possible constant $\lambda_{2}=f_{a_{2}}(3 / 2)=0.009063 \ldots$.

Remark 2 The upper bound for $R_{n}-\gamma$ given in (3.2) is better than that given in (1.5). Indeed, simple computations show that

$$
\begin{aligned}
& \frac{1}{24} \frac{\left(n+\frac{1}{2}\right)^{2}-\frac{7}{40}}{\left(n+\frac{1}{2}\right)^{4}-\frac{31}{336}}-\left(\frac{1}{24 n^{2}}+\frac{7}{960} \frac{1}{n^{4}}\right) \\
& \quad=-\frac{6,720 n^{5}+10,752 n^{4}+5,712 n^{3}+1,564 n^{2}+588 n-35}{960 n^{4}\left(168 n^{4}+336 n^{3}+252 n^{2}+84 n-5\right)}<0
\end{aligned}
$$

for all $n \in \mathbb{N}$.

Theorem 4 Let $R(x)$ be defined on $(0, \infty)$ by $(1.10)$. Then the function

$$
x \mapsto G_{a}(x)=24\left(x^{4}+a x^{2}+\frac{7}{40} a-\frac{31}{336}\right) R(x)-\left(x^{2}-\frac{7}{40}+a\right)
$$

is strictly completely monotonic on $(0, \infty)$ if and only if $a \geq a_{3}=11,165 / 8,284$. 
Proof The necessity can be derived from

$$
\begin{aligned}
\lim _{x \rightarrow \infty} \frac{G_{a}(x)}{x^{-4}} & =\lim _{x \rightarrow \infty} \frac{24\left(x^{4}+a x^{2}+\frac{7}{40} a-\frac{31}{336}\right)(\psi(x+1 / 2)-\ln x)-\left(x^{2}-\frac{7}{40}+a\right)}{x^{-4}} \\
& =\frac{2,071}{33,600}\left(a-\frac{11,165}{8,284}\right) \geq 0 .
\end{aligned}
$$

It follows from (2.2), (2.4), and (2.6) that

$$
\begin{aligned}
G_{a}(x)= & 24 x^{4} R(x)+24 a x^{2} R(x)+24\left(\frac{7}{40} a-\frac{31}{336}\right) R(x)-\left(x^{2}-\frac{7}{40}+a\right) \\
= & 24\left(\frac{1}{24} x^{2}-\frac{7}{960}+\int_{0}^{\infty} e^{-x t} Q^{(4)}(t) d t\right)+24 a\left(\frac{1}{24}+\int_{0}^{\infty} e^{-x t} Q^{\prime \prime}(t) d t\right) \\
& +24\left(\frac{7}{40} a-\frac{31}{336}\right) \int_{0}^{\infty} e^{-x t} Q(t) d t-\left(x^{2}-\frac{7}{40}+a\right) \\
= & 24 \int_{0}^{\infty} e^{-x t}\left(Q^{(4)}(t)+a Q^{\prime \prime}(t)+\left(\frac{7}{40} a-\frac{31}{336}\right) Q(t)\right) d t \\
\triangleq & 24 \int_{0}^{\infty} e^{-x t} g_{a}(t) d t .
\end{aligned}
$$

From Lemmas 5 and 7 we clearly see that

$$
\begin{aligned}
g_{a}(t) & =Q^{(4)}(t)-\frac{31}{336} Q(t)+a\left(Q^{\prime \prime}(t)+\frac{7}{40} Q(t)\right) \\
& \geq Q^{(4)}(t)-\frac{31}{336} Q(t)+\frac{11,165}{8,284}\left(Q^{\prime \prime}(t)+\frac{7}{40} Q(t)\right) \\
& =Q^{(4)}(t)+\frac{11,165}{8,284} Q^{\prime \prime}(t)+\frac{199,849}{1,391,712} Q(t)>0
\end{aligned}
$$

if $a \geq a_{3}=11,165 / 8,284$.

The monotonicity of $G_{a_{3}}$ and the facts that

$$
\begin{aligned}
& G_{a_{3}}\left(\frac{3}{2}\right)=\frac{112,672,809}{579,880}-\frac{11,465,761}{57,988} \ln \frac{3}{2}-\frac{11,465,761}{57,988} \gamma=0.001690 \ldots, \\
& G_{a_{3}}(\infty)=0
\end{aligned}
$$

lead to the following.

Corollary 4 Let $R_{n}$ be defined by (1.3). Then the double inequality

$$
\begin{aligned}
& \frac{1}{24} \frac{\left(n+\frac{1}{2}\right)^{2}+\frac{97,153}{82,840}}{\left(n+\frac{1}{2}\right)^{4}+\frac{11,165}{8,284}\left(n+\frac{1}{2}\right)^{2}+\frac{199,849}{1,391,712}} \\
& \quad<R_{n}-\gamma<\frac{1}{24} \frac{\left(n+\frac{1}{2}\right)^{2}+\frac{97,153}{82,840}+\lambda_{3}}{\left(n+\frac{1}{2}\right)^{4}+\frac{11,165}{8,284}\left(n+\frac{1}{2}\right)^{2}+\frac{199,849}{1,391,712}}
\end{aligned}
$$

holds for all $n \in \mathbb{N}$ with the best possible constant $\lambda_{3}=G_{a_{3}}(3 / 2)=0.001690 \ldots$. 


\section{Remarks}

Remark 3 The function $G_{a}$ defined by (3.3) can be rewritten as

$$
G_{a}(x)=a \times f_{7 / 40}(x)-F_{-31 / 336}(x)=f_{7 / 40}(x) \times\left(a-\frac{F_{-31 / 336}(x)}{f_{7 / 40}(x)}\right) .
$$

Theorem 4 leads to the conclusion that

$$
\frac{F_{-31 / 336}(x)}{f_{7 / 40}(x)} \leq \lim _{x \rightarrow \infty} \frac{F_{-31 / 336}(x)}{f_{7 / 40}(x)}=\lim _{x \rightarrow \infty} \frac{-24\left(x^{4}-\frac{31}{336}\right) R(x)+x^{2}-\frac{7}{40}}{24\left(x^{2}+\frac{7}{40}\right) R(x)-1} \leq \frac{11,165}{8,284} .
$$

Moreover, we can prove that

$$
\frac{F_{-31 / 336}(x)}{f_{7 / 40}(x)} \geq \lim _{x \rightarrow 0^{+}} \frac{F_{-31 / 336}(x)}{f_{7 / 40}(x)}=\lim _{x \rightarrow 0^{+}} \frac{-24\left(x^{4}-\frac{31}{336}\right) R(x)+x^{2}-\frac{7}{40}}{24\left(x^{2}+\frac{7}{40}\right) R(x)-1} \geq \frac{155}{294} .
$$

It suffices to prove the function

$$
x \mapsto V(x)=\psi(x+1 / 2)-\ln x-\frac{1}{24} \frac{x^{2}+\frac{2,071}{5,880}}{x^{2}\left(x^{2}+\frac{155}{294}\right)}
$$

is increasing on $(0, \infty)$. Differentiation gives

$$
V^{\prime}(x)=\psi^{\prime}(x+1 / 2)-\frac{1}{x}+\frac{1,728,720 x^{4}+1,217,748 x^{2}+321,005}{10 x^{3}\left(294 x^{2}+155\right)^{2}} .
$$

From $\psi^{\prime}(x+1)-\psi^{\prime}(x)=-1 / x^{2}$ we get

$$
\begin{aligned}
& V^{\prime}(x+1)-V^{\prime}(x) \\
&=- \frac{1}{(x+1 / 2)^{2}}-\frac{1}{x+1}+\frac{1,728,720(x+1)^{4}+1,217,748(x+1)^{2}+321,005}{10(x+1)^{3}\left(294(x+1)^{2}+155\right)^{2}} \\
&+\frac{1}{x}-\frac{1,728,720 x^{4}+1,217,748 x^{2}+321,005}{10 x^{3}\left(294 x^{2}+155\right)^{2}} \\
&=-\frac{V_{1}(x)}{10 x^{3}(2 x+1)^{2}\left(294 x^{2}+155\right)^{2}(x+1)^{3}\left(294 x^{2}+588 x+449\right)^{2}},
\end{aligned}
$$

where

$$
\begin{aligned}
V_{1}(x)= & 1,718,371,882,080 x^{12}+10,310,231,292,480 x^{11} \\
& +29,399,355,669,600 x^{10}+52,486,324,833,600 x^{9} \\
& +66,690,983,696,400 x^{8}+65,258,530,001,280 x^{7} \\
& +51,909,045,513,612 x^{6}+34,352,301,620,196 x^{5} \\
& +18,881,999,450,054 x^{4}+8,378,736,976,048 x^{3} \\
& +2,808,871,359,013 x^{2}+622,502,847,155 x \\
& +64,714,929,005>0
\end{aligned}
$$


for $x>0$. Therefore,

$$
V^{\prime}(x)>V^{\prime}(x+1)>V^{\prime}(x+2)>\cdots>\lim _{n \rightarrow \infty} V^{\prime}(x+n)=0
$$

for all $x>0$.

In addition, (4.1) implies that the necessary condition such that the function $-G_{a}$ is completely monotone on $(0, \infty)$ is

$$
a \leq \lim _{x \rightarrow 0+} \frac{F_{-31 / 336}(x)}{f_{7 / 40}(x)}=\lim _{x \rightarrow 0+} \frac{-24\left(x^{4}-\frac{31}{336}\right) R(x)+x^{2}-\frac{7}{40}}{24\left(x^{2}+\frac{7}{40}\right) R(x)-1}=\frac{155}{294}
$$

Motivated by inequalities (4.2) and (4.3) we propose two conjectures.

Conjecture 1 Let $R(x)$ be defined on $(0, \infty)$ by (1.10). Then we conjecture that

(i) the function

$$
x \mapsto \frac{-24\left(x^{4}-\frac{31}{336}\right) R(x)+x^{2}-\frac{7}{40}}{24\left(x^{2}+\frac{7}{40}\right) R(x)-1}
$$

is increasing on $(0, \infty)$;

(ii) the function $-G_{a}$ is completely monotone on $(0, \infty)$ if and only if $a \leq 155 / 294$.

Remark 4 The monotonicity of the function $V$ proved in Remark 3 and the facts that

$$
V\left(\frac{3}{2}\right)=\frac{866,519}{881,820}-\ln \frac{3}{2}-\gamma=-0.00003238 \ldots, \quad V(\infty)=0
$$

lead to the conclusion that the double inequality

$$
\frac{1}{24} \frac{\left(n+\frac{1}{2}\right)^{2}+\frac{2,071}{5,880}}{\left(n+\frac{1}{2}\right)^{2}\left(\left(n+\frac{1}{2}\right)^{2}+\frac{155}{294}\right)}+\lambda_{4}<R_{n}-\gamma<\frac{1}{24} \frac{\left(n+\frac{1}{2}\right)^{2}+\frac{2,071}{5,880}}{\left(n+\frac{1}{2}\right)^{2}\left(\left(n+\frac{1}{2}\right)^{2}+\frac{155}{294}\right)}
$$

holds with the best possible constant $\lambda_{4}=-0.00003238 \ldots$

The upper bound for $R_{n}-\gamma$ in (4.4) is better than that in (3.2) because of

$$
\begin{aligned}
& \frac{1}{24} \frac{\left(n+\frac{1}{2}\right)^{2}+\frac{2,071}{5,880}}{\left(n+\frac{1}{2}\right)^{2}\left(\left(n+\frac{1}{2}\right)^{2}+\frac{155}{294}\right)}-\frac{1}{24} \frac{\left(n+\frac{1}{2}\right)^{2}-\frac{7}{40}}{\left(n+\frac{1}{2}\right)^{4}-\frac{31}{336}} \\
& \quad=-\frac{64,201}{120(2 n+1)^{2}\left(588 n^{2}+588 n+457\right)\left(168 n^{4}+336 n^{3}+252 n^{2}+84 n-5\right)}<0 .
\end{aligned}
$$

\section{Remark 5 Let}

$$
\begin{aligned}
& w_{n}=\sum_{k=1}^{n} \frac{1}{k}-\ln (n+1 / 2)-\frac{1}{24} \frac{\left(n+\frac{1}{2}\right)^{2}-\frac{7}{40}}{\left(n+\frac{1}{2}\right)^{4}-\frac{31}{336}}, \\
& y_{n}=\sum_{k=1}^{n} \frac{1}{k}-\ln (n+1 / 2)-\frac{1}{24} \frac{\left(n+\frac{1}{2}\right)^{2}+\frac{97,153}{82,840}}{\left(n+\frac{1}{2}\right)^{4}+\frac{11,165}{8,284}\left(n+\frac{1}{2}\right)^{2}+\frac{199,849}{1,391,712}}, \\
& z_{n}=\sum_{k=1}^{n} \frac{1}{k}-\ln (n+1 / 2)-\frac{1}{24} \frac{\left(n+\frac{1}{2}\right)^{2}+\frac{2,071}{5,880}}{\left(n+\frac{1}{2}\right)^{2}\left(\left(n+\frac{1}{2}\right)^{2}+\frac{155}{294}\right)} .
\end{aligned}
$$


Then Theorems 3 and 4 together with Remark 4 lead to

$$
w_{n}<z_{n}<\gamma<y_{n}
$$

and simple computations show that

$$
\begin{aligned}
& \lim _{n \rightarrow \infty} n^{8}\left(w_{n}-\gamma\right)=-\frac{319}{92,160}, \\
& \lim _{n \rightarrow \infty} n^{10}\left(y_{n}-\gamma\right)=\frac{627,404,761}{246,900,842,496}, \\
& \lim _{n \rightarrow \infty} n^{8}\left(z_{n}-\gamma\right)=-\frac{199,849}{94,832,640} .
\end{aligned}
$$

Lastly, inspired by Theorems 2-4, we propose an open problem as follows.

Problem 1 We wonder what the sequences $\left\{a_{k}\right\}$ and $\left\{b_{k}\right\}$ are such that the function

$$
x \mapsto R(x) \sum_{k=0}^{n+1} a_{k} x^{2 k}-\sum_{k=0}^{n} b_{k} x^{2 k}
$$

is completely monotone on $(0, \infty)$ and

$$
\lim _{x \rightarrow \infty} \frac{R(x) \sum_{k=0}^{n+1} a_{k} x^{2 k}-\sum_{k=0}^{n} b_{k} x^{2 k}}{x^{-2 n-4}}=c \neq 0, \pm \infty .
$$

\section{Competing interests}

The authors declare that they have no competing interests.

\section{Authors' contributions}

All authors contributed equally to the writing of this paper. All authors read and approved the final manuscript.

\section{Acknowledgements}

This research was supported by the Natural Science Foundation of China under Grants 11401191 and 61374086, and the Natural Science Foundation of Zhejiang Province under Grant LY13A010004

Received: 5 January 2015 Accepted: 22 April 2015 Published online: 12 May 2015

\section{References}

1. Widder, DV: The Laplace Transform. Princeton University Press, Princeton (1941)

2. Rippon, PJ: Convergence with pictures. Am. Math. Mon. 93(6), 476-478 (1986)

3. Young, RM: Euler's constant. Math. Gaz. 75(422), 187-190 (1991)

4. Tims, SR, Tyrrell, JA: Approximate evaluation of Euler's constant. Math. Gaz. 55(371), 65-67 (1971)

5. Tóth, L: Problem E3432. Am. Math. Mon. 98(3), 264 (1991)

6. Tóth, L, High, R, Knuth, DE, Graham, RL, Patashnik, O: Problems and solutions: solutions: E3432. Am. Math. Mon. 99(7), 684-685 (1992)

7. DeTemple, DW: A quicker convergence to Euler's constant. Am. Math. Mon. 100(5), 468-470 (1993)

8. Negoi, T: A faster convergence to the constant of Euler. Gaz. Mat., Ser. A 15, 111-113 (1997)

9. Vernescu, A: A new accelerate convergence to the constant of Euler. Gaz. Mat., Ser. A 17, 273-278 (1999)

10. Qi, F, Cui, R-Q, Chen, C-P, Guo, B-N: Some completely monotonic functions involving polygamma functions and an application. J. Math. Anal. Appl. 310(1), 303-308 (2005)

11. Sîntămărian, A: A generalization of Euler's constant. Numer. Algorithms 46(2), 141-151 (2007)

12. Chen, C-P, Qi, F: The best bounds of the $n$-th harmonic number. Glob. J. Appl. Math. Math. Sci. 1(1), $41-49$ (2008)

13. Sîntămărian, A: Some inequalities regarding a generalization of Euler's constant. JIPAM. J. Inequal. Pure Appl. Math. 9(2), Article 46 (2008)

14. Villarino, MB: Ramanujan's harmonic number expansion into negative powers of triangular number. JIPAM. J. Inequal. Pure Appl. Math. 9(3), Article 89 (2008)

15. Chen, C-P: Inequalities and monotonicity properties for some special functions. J. Math. Inequal. 3(1), 79-91 (2009)

16. Chen, C-P: The best bounds in Vernescu's inequalities for the Euler's constant. RGMIA Res. Rep. Collect. 12(3), Article ID 11 (2009). http://ajmaa.org/RGMIA/v12n3.php 
17. Chen, C-P: Monotonicity properties of functions related to the psi function. Appl. Math. Comput. 217(7), 2905-2911 (2010)

18. Chen, C-P: Inequalities for the Euler-Mascheroni constant. Appl. Math. Lett. 23, 161-164 (2010)

19. Mortici, C: On new sequences converging towards the Euler-Mascheroni constant. Comput. Math. Appl. 59(8), 2610-2614 (2010)

20. Mortici, C: Improved convergence towards generalized Euler-Mascheroni constant. Appl. Math. Comput. 215(9), 3443-3448 (2010)

21. Mortici, C: Fast convergences towards Euler-Mascheroni constant. Comput. Appl. Math. 29(3), 479-491 (2010)

22. Guo, B-N, Qi, F: Sharp bounds for harmonic numbers. Appl. Math. Comput. 218(3), 991-995 (2011)

23. Chen, C-P: Sharpness of Negoi's inequality for the Euler-Mascheroni constant. Bull. Math. Anal. Appl. 3(1), 134-141 (2011)

24. Mortici, C: A new Stirling series as continued fraction. Numer. Algorithms 56(1), 17-26 (2011)

25. Chen, C-P, Mortici, C: New sequence converging towards the Euler-Mascheroni constant. Comput. Math. Appl. 64(4), 391-398 (2012)

26. Gavrea, I, Ivan, M: A solution to an open problem on the Euler-Mascheroni constant. Appl. Math. Comput. 224, 54-57 (2013)

27. Lu, D: Some new convergent sequences and inequalities of Euler's constant. J. Math. Anal. Appl. 419(1), 541-552 (2014)

28. Mortici, C: New bounds for a convergence by DeTemple. J. Sci. Arts 13(2), 239-242 (2010)

29. Batir, N: Inequalities for the gamma function. Arch. Math. 91(6), 554-563 (2008)

30. Qi, F, Guo, B-N: Sharp inequalities for the psi function and harmonic numbers (2009). arXiv:0902.2524 [math. CA]

31. Guo, B-N, Qi, F: Sharp inequalities for the psi function and harmonic numbers. Analysis 34(2), 201-208 (2014)

32. Karatsuba, EA: On the computation of the Euler constant $\gamma$. Numer. Algorithms 24(1-2), 83-97 (2000)

33. Anderson, GD, Vamanamurthy, MK, Vuorinen, M: Topics in special functions. In: Papers on Analysis. Rep. Univ. Jyväskylä Dep. Math. Stat., vol. 83, pp. 5-26. Univ. Jyväskylä, Jyväskylä (2001)

34. Abramowitz, M, Stegun, IA: Handbook of Mathematical Functions with Formulas, Graphs, and Mathematical Tables. U.S. Government Printing Office, Washington (1964)

35. Yang, Z-H, Chu, Y-M, Tao, X-J: A double inequality for the trigamma function and its applications. Abstr. Appl. Anal. 2014, Article ID 702718 (2014)

36. Yang, Z-H, Chu, Y-M: A note on Jordan, Adamović-Mitrinović, and Cusa inequalities. Abstr. Appl. Anal. 2014, Article ID 364076 (2014)

\section{Submit your manuscript to a SpringerOpen ${ }^{\ominus}$ journal and benefit from:}

- Convenient online submission

Rigorous peer review

- Immediate publication on acceptance

- Open access: articles freely available online

- High visibility within the field

- Retaining the copyright to your article 